\title{
HISTORIA DEL DERECHO NATURAL Y DE GENTES
}

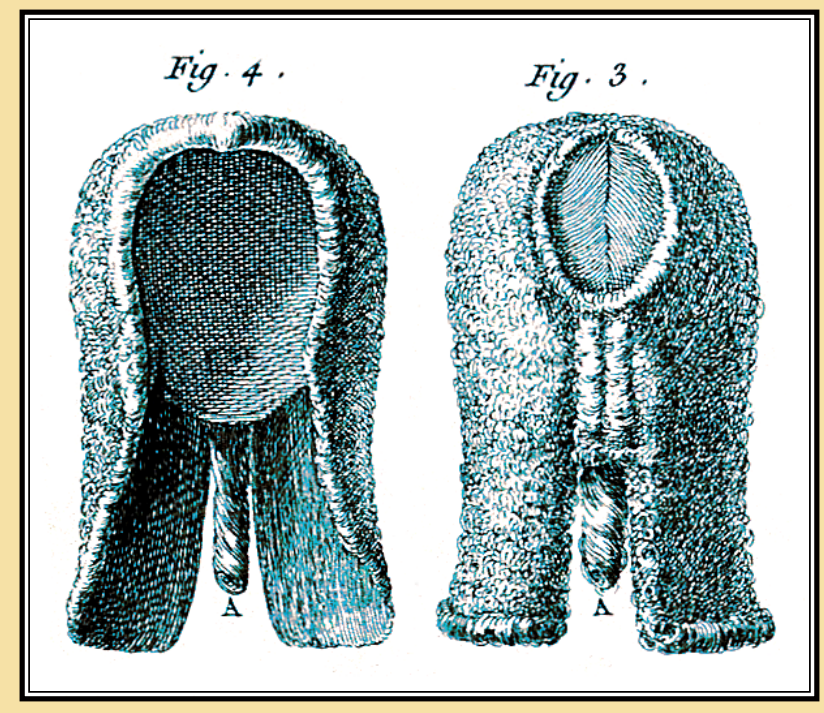

JoAQuín Marín y Mendoza 

Historia del derecho natural y de gentes 
The Figuerola Institute

Programme: Legal History

The Programme "Legal History" of the Figuerola Institute of Social Science History -a part of the Carlos III University of Madrid- is devoted to improve the overall knowledge on the history of law from different points of view -academically, culturally, socially, and institutionally- covering both ancient and modern eras. A number of experts from several countries have participated in the Programme, bringing in their specialized knowledge and dedication to the subject of their expertise.

To give a better visibility of its activities, the Programme has published in its Book Series a number of monographs on the different aspects of its academic discipline.

Publisher:

Carlos III University of Madrid

Book Series:

Legal History

Editorial Committee:

Manuel Ángel Bermejo Castrillo, Universidad Carlos III de Madrid

Catherine Fillon, Université Jean Moulin Lyon 3

Manuel Martínez Neira, Universidad Carlos III de Madrid

Carlos Petit, Universidad de Huelva

Cristina Vano, Università degli studi di Napoli Federico II

More information at www.uc3m.es/legal_history 
Historia del derecho natural y de gentes

Joaquín Marín y Mendoza

UNIVERSIDAD CARLOS III DE MADRID

2015 
Historia del derecho, 39

(C) 2015 Manuel Martínez Neira

Preimpresión: TALLERONCE

ISBN: 978-84-89315-91-4

ISSN: $2255-5137$

Versión electrónica disponible en e-Archivo

http://hdl.handle.net/10016/22079

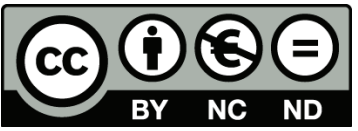

Licencia Creative Commons Atribución-NoComercial-SinDerivadas 3.o España 


\section{ÍNDICE}

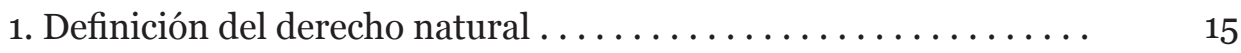

2. Definición del derecho de gentes ...................... $\quad 15$

3. Diferencia de otras ciencias . . . . . . . . . . . . . . . . . 16

4. Del derecho público $\ldots \ldots \ldots \ldots \ldots \ldots \ldots \ldots \ldots \ldots \ldots \ldots$

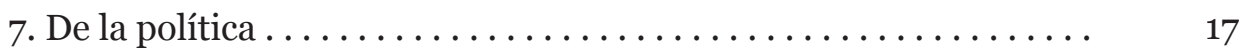

9. Necesidad y utilidad del derecho natural y de gentes .......... 19

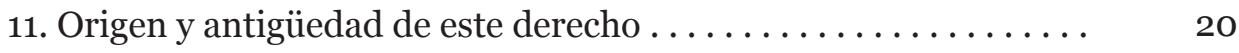

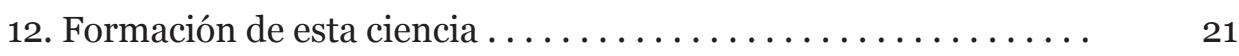

13. Progresos y escritores principales. Hugo Grotius ........... 23

17. John Selden .............................. $\quad 26$

18. Thomas Hobbes............................ $\quad 26$

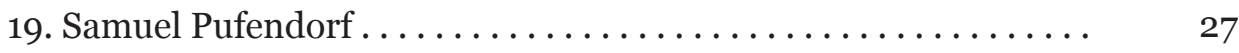

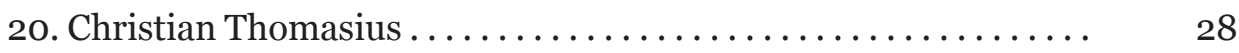

21. Johann Gottlieb Heineccius . . . . . . . . . . . . . . . . . . 29

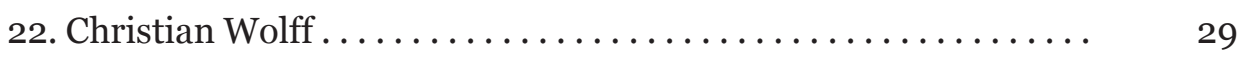

23. Emer de Vattel ............................... 30

24. Jean-Jacques Burlamaqui. Fortunato Bartolomeo De Felice .... 31

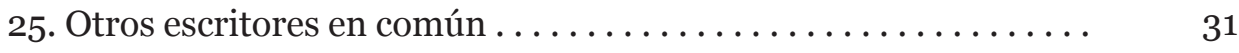

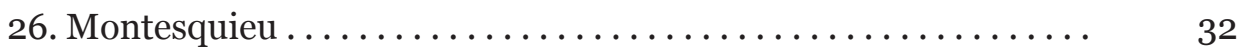

27. Simon-Nicolas-Henri Linguet ...................... 33

28. Jean-Jacques Rousseau .......................... 33

29. Escritos modernos detestables . .................... 33

30. Vicios y defectos de muchos modernos ................ 34

31. Modo para conocer los autores sospechosos ............. $\quad 35$

32. Autores católicos con que se refutan ................. $\quad 35$

33. Precaución para establecer las reglas del derecho natural ..... $\quad 36$

34. Falsa preocupación de buscar un primer principio .......... 37

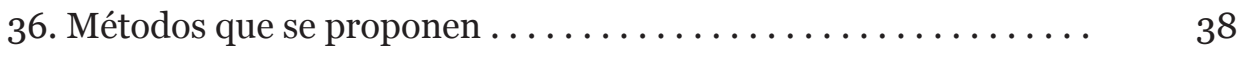

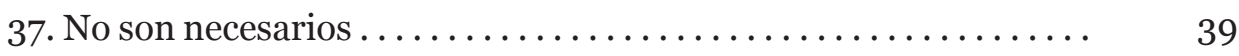

38. Del método escolástico .......................... 39

39. Universidades en que se enseña este derecho ............. 40 

Joaquín Marín y Mendoza (1727-1782) fue abogado de los Reales Consejos, académico de la Historia y alcalde del Crimen. Aquí nos interesa sobre todo por haber ocupado la cátedra de derecho natural que se erigió en los Reales Estudios de San Isidro, en Madrid. A él se debe una edición del manual de Heineccius para esta materia: Elementa Juris Naturae et Gentium, castigationibus ex Catholicorum doctrina, Madrid 1776.

A continuación, en ese mismo año, publicó su Historia del derecho natural y de gentes, la obra que ahora se edita y que, en sus palabras, constituía una guía para un acercamiento sistemático a esa ciencia. Es decir, no era una historia literaria (de autores y obras) sino una primera lección o introducción para el que emprendía este estudio, aunque fuese de manera personal.

Como ya indicó Manuel García-Pelayo, al publicarla (Madrid 1950), la obra interesa sobre todo como "exponente de una situación que predominaba en los círculos dirigentes y en las capas cultas de la España del siglo XVIII con respecto al derecho natural racionalista" (p. 7); un derecho que había "seducido" al autor y a la época (pp. 12-13). La situación o disposición referida se deduce rápidamente de la aparente contradicción que encontramos al recorrer los márgenes del discurso: necesidad y utilidad del derecho natural, escritos modernos detestables, vicios y defectos de muchos modernos...

Una y otra obra, Elementa e Historia, al compartir un mismo objetivo expurgatorio, se complementan. Así lo indica el autor en el párrafo 32 (página 36 de esta edición), al manifestar la mira con la que había hecho la edición del libro de Heineccius.

Además de la edición original y la ya mencionada de García-Pelayo, tenemos otra de Salvador Rus Rufino (València 1999). La presente parte del texto de 1776, al que quiere ser fiel. El autor dividió su discurso en cuarenta párrafos, división que aquí se respeta, algunos carecen de epígrafe (márgenes) y otros tienen dos. Para facilitar la lectura, se ha modernizado la puntuación y la ortografía (método por methodo, por ejemplo), pero se conserva la de los nombres propios. Se han completado las notas al pie, lo imprescindible para facilitar la localización de las referencias, y se han corregido cuando era necesario (Giovanni Francesco Finetti en vez de Juan Bautista Fineti, por ejemplo).

M. M. N. 



\section{HISTORIA \\ DEL DERECHO NATURAL \\ Y DE GENTES}

Por Don Joaquín Marín

Abogado de los Reales Consejos, del Ilustre

Colegio, y Catedrático de este mismo

Derecho en los Reales Estudios

de esta Corte

Madrid 1776

Por D. Manuel Martín, calle de la Cruz, donde se hallará

Con las licencias necesarias 



\section{TABLA}

Definición del Derecho Natural

$\S 1$

Definición del Derecho de Gentes

$\S 2$

Diferencia de otras Ciencias

$\S 3$

Del Derecho Público

De la Política

Necesidad y utilidad del Derecho Natural y de Gentes

Origen y antigüedad de este Derecho

Formación de esta Ciencia

Progresos y Escritores principales

Hugo Grocio

$\S 13$

Juan Seldeno

Thomas Hobbes

$\S 18$

Samuel Puffendorf

Christiano Thomasio

$\S 20$

Juan Gottlieb Heineccio

$\S 21$

Christiano Wolfio

$\S 22$

Wattel

$\S 23$

Burlamaqui

$\S 24$

Felice

$\S 24$

Otros Escritores en común

$\S 25$

Montesquieu

$\S 26$

Linguet

Rousseau

$\S 28$

Escritos modernos detestables

Vicios y defectos de muchos modernos

Modo para conocer los Autores sospechosos

Autores Católicos con que se refutan

Precaución para establecer las reglas del Derecho Natural

Falsa preocupación de buscar un primer principio

Métodos que se proponen

$\S 36$

No son necesarios

Del método Escolástico

Universidades en que se enseña este Derecho 

Lleva este corto Discurso el título de Historia de el Derecho Natural y de Gentes porque trata de su origen y progreso; de los Autores y de algunas doctrinas más particulares de ellos; de los principales sistemas y métodos; de las preocupaciones y vicios de que conviene librarse; y de los medios y preocupaciones necesarias con que se debe proceder. Podía tener el asunto otra extensión mucho más dilatada y, tanto por esto como porque la materia que se ha elegido se ofrece con mucha concisión, más bien pedía que se le diese el nombre de Epítome que de Historia. Pero como esto último tiene más dificultad de lo que a la vista se presenta y no sea tan conducente al fin que se ha propuesto, que es solo dejar una guía segura a los que toman los primeros elementos de esta ciencia para que se puedan ordenar por sí después un estudio bien que privado más formal; por este motivo no se deberá culpar que se atribuya el nombre todo entero a la parte más principal y noble de la materia, como suele comúnmente suceder.

1

\section{Definición del Derecho Natural}

Por Derecho Natural se entiende un conjunto de leyes dimanadas de Dios y participadas a los hombres por medio de la razón natural; los cuales, teniendo con esta luz bastante auxilio para alcanzar sus más principales preceptos, están obligados por lo mismo a regular según ellos sus acciones. Llámase Natural porque no le forman los hombres, sino que le dio y enseñó a todos el Autor de la naturaleza, ordenando, por medio de sus principios y máximas, la constitución admirable y prodigiosa armonía de todo el género humano. Pues así como se llama natural en el hombre la locución, la risa, el oído, la inteligencia, porque la misma naturaleza le va suministrando estas facultades desde que nace, del mismo modo, se llama Derecho Natural el que lleva el hombre grabado, como dice el Apóstol, en el corazón, desde su cuna, para el gobierno de su vida.

2

\section{Definición del Derecho de Gentes}

Por el Derecho de Gentes queremos igualmente denotar aquellos mismos primeros principios que el Derecho Natural establece, en cuanto se aplican para 
regir y gobernar los mutuos oficios y negocios de los Estados y naciones; las leyes que éstos mismos se imponen por medio de los pactos y tratados, y las máximas que su propio bien y felicidad precisa recibir. Llámase Derecho de Gentes, porque esta palabra, comúnmente, se toma en esta ciencia por toda nación o Estado político, que no es otro que una gran porción de gentes y familias reunidas.

3

\section{Diferencia de otras Ciencias}

Esta ciencia no es lo mismo que la del Derecho Público y la Política, y conviene que mostremos la notable diferencia que media entre las tres; así porque el distinguir las cosas que entre sí parecen semejantes es medio para conocer el constitutivo peculiar de cada una, como porque, de este modo, se evitará la confusión que comúnmente se hace llamando, con equivocación voluntaria, a esta enseñanza del Derecho Público.

4

Del Derecho Público

Son varias las significaciones que se dan por los autores a estas dos palabras: Derecho Público. Entre ellos, unas veces se llama público el Derecho por la forma y causa eficiente, esto es, con respeto a la autoridad soberana que lo establece, a diferencia de aquel que cada uno se adquiere por medio de los pactos. En otras ocasiones se toma por el que es común y compete a todos los de una República, mientras otros son sólo peculiares y privativos de algunas personas. Y, por fin, con este nombre suelen indicar un derecho que es público por su objeto y materia, pues prescribe lo que es útil a todo pueblo y Estado junto, prescindiendo de los individuos a quienes no alcanza sino por la resultancia y consecuencia, porque es preciso difunda beneficio a los miembros lo que nutre y utiliza a todo el cuerpo.

Esta es la noción más propia con que comúnmente se suele tomar y con la que hablamos al presente, en cuyo sentido es en rigor el que descubre la organización de cada Estado; el modo con que en su principio se forma; las clases que 
tiene de vasallos, sus derechos y prerrogativas; aquel o aquellos en quienes reside el poder soberano; el modo cómo lo ejerce en la administración de Justicia, en la Hacienda y demás partes de que se compone la Majestad, tanto en tiempo de paz como de guerra, según las leyes fundamentales de cada nación.

Todos estos diversos puntos forman un cuerpo de conocimientos o sistema político que varía con respecto a la nación de que se habla. Por lo mismo, se divide el Derecho Público en universal y particular. Aquél se ocupa en dar las reglas y principios generales con que se debe componer cada Estado para ser perfecto, según las diferentes especies y formas de República. El particular indaga la interior disposición de este o el otro Estado determinado, y así, es muy diferente el Derecho Público de España del de Francia, Inglaterra y otros países, al modo que son muy distintos sus gobiernos. Heineccio, uno de los autores de que usamos por ahora en esta enseñanza, delineando, en el libro segundo de los elementos del Derecho Natural, la estructura propia de todos los Estados, con razón dijo que entonces trataba del Derecho Público Univer$\mathrm{sal}^{1}$. Quum ergo hic non sit quaestio de Jure clientelari, sed publico universali... Y el padre Francisco Schmier dio el título de Jurisprudencia Pública Universal a la obra que salió a luz en 1728, trazada sobre este plan mismo. Por el contrario, el señor don Pedro Josef Pérez Valiente, hoy del Supremo Consejo de Castilla, como se propuso por objeto la constitución de nuestra Monarquía, con razón llamó Aparato del Derecho Público Español a los dos tomos que imprimió en 1751, donde, después de haber juntado con mucha doctrina los materiales sobre que debía apoyar esta gran masa, empezó a echar los primeros cimientos a un edificio que no es poco difícil de concluir. Basten para noticia estos ejemplares que son conocidos entre nosotros.

\section{De la Política}

La Política supone las sociedades civiles o Estados, la autoridad, las leyes y todos los demás objetos sobre que versa el Derecho Público, y pasa, supuestos estos antecedentes, a examinar la forma de gobierno que es más conveniente y los medios que más conducen a que el pueblo viva tranquilo y con todo lo

1 Heineccius, Elementa juris naturae et gentium, libro 2. § 135. schol. 
necesario para su conservación y mayor comodidad. Así, es muy diferente su destino y meditación, pero, sin embargo, se confunde comúnmente con el Derecho Público, sino es en aquellos países donde su constitución precisa a cultivar más que en otros esta ciencia; como sucede en Alemania, que, como es una República irregular, compuesta de tantos príncipes y pequeños Estados y señoríos, por lo mismo ocurren tantas controversias sobre el Derecho Público como sobre el Civil y Privado. Por lo que decía muy bien Struvio²: Nulla Respublica prolixiori utitur jure publico, quan nostra Germania, eo quod legibus publicis abundat, libertateque eximia gaudet. Nec usitatum facile in aliis rebuspublicis nomen est juris publici, quod apud Germanos ideo receptum, quod ejusdem doctrinam jureconsulti sibi vindicent, cum in aliis regnis Notitia Status Politici potissimum dicatur.

Bien claro está, por lo dicho, cuán distintas son éstas cosas entre sí; y cómo de cosas diversas hace un retrato de cada una, con vivos colores, el sabio Abad Claudio Fleuri en su preciosa obra del Método de los Estudios, del que no me quiero privar porque, al mismo tiempo que enseña haciendo un cotejo entre las dos, se extiende a dar muy prudentes y oportunos consejos. Este escritor en el capítulo veinte y seis, que intitula de la Política habla de este modo a los jóvenes de distinguido nacimiento: "Quisiera yo darles a conocer, en primer lugar, el estado del Gobierno presente de su país, las diferentes partes de que está compuesto este cuerpo, el modo de hacer justicia, de administrar la hacienda, de ejercer la política y así de los demás, la forma de los consejos para los negocios públicos. Querría que comenzasen por el estado de su país, como el más necesario y más fácil de conocer; después se alargasen a los países extranjeros, los más cercanos y con quienes hay mayor conexión. En habiéndoles mostrado cómo son en efecto las cosas, les mostraría cómo deben ser, no según la opinión de los filósofos y el puro discurso, sino según las leyes del mismo Estado y sus antiguos usos. Llamo Derecho Público las reglas con las cuales se gobierna cada Estado, los derechos del soberano y de los oficiales de que se sirve, los derechos de los Estados y de los soberanos, unos respecto de otros. Este estudio es más positivo que de discurso y encierra en sí mucha historia que puede hacerlo agradable.” Después prosigue explicándose sobre la Política en estos términos: "La Política consiste más en discurso y debe

2 Struvius, Bibliotheca juris selecta, cap. 57 , § 5 . 
subir más alto en la investigación de los principios. No mira ésta sólo cómo deben ser gobernadas España, Francia o Alemania; según la forma particular de su Estado y las leyes que se hallan establecidas en ellas, considera en general lo que es la sociedad civil, cuál forma de Estado es la mejor, cuáles son las mejores leyes y los mejores modos de mantener la tranquilidad y la unión entre los hombres. Estas consideraciones generales son más útiles para dar al entendimiento elevación y extensión, con tal que se haga aplicación de ellas sobre ejemplos particulares y no se contenten con los antiguos de Atenas y Lacedemonia, sino que se tomen los modernos, que nos importan e instruyen mejor. La advertencia que me parece más importante en esta materia es dar a conocer con tiempo a un príncipe mozo, o a cualquier otro niño, la diferencia de la verdadera y de la falsa Política. Que tome horror a lo que tiene sólo por fin hacer poderoso al príncipe, o al cuerpo que gobierna, a costa de todo lo restante del pueblo. A que no ponga la virtud del soberano en mantener y aumentar su poder dejando a los particulares la justicia, la fidelidad y la humanidad."

9

\section{Necesidad y utilidad del Derecho Natural y de Gentes}

Nuestro asunto no es el Derecho Público, ni la Política, sino el Derecho Natural y de Gentes. Hablamos aquí de aquellas reglas que tienen prescritas los hombres para ajustar sus acciones, ya se les considere privadamente de unos a otros, ya como unidos en cuerpos y sociedades. Estando dotados de inteligencia y razón, pueden conocer las cosas y comparar las presentes con las pasadas para discernir después entre ellas; son capaces de formar juicio en virtud de la misma potencia, de suspenderlo, como también de reflexionar y elegir unas y desechar otras; las cuales dotes serían del todo inútiles si, quedando ciegos con el primer impulso que causan los objetos, fuesen de tropel arrebatados por su fuerza. Por otra parte, las mismas prerrogativas con que se aventaja a las demás criaturas piden que obre conforme a su naturaleza racional y que, escogiendo sólo las acciones que merecen premio y alabanza, se haga digno de tan singular beneficio. Su talento propio le fortifica más en los preceptos de la religión porque halla dentro de sí mismo el convencimiento de una verdad cuya ignorancia le fuera por todos términos inexcusable. Conoce el arte y método con que los hombres, guiados de la sola luz natural, se han puesto justos límites en las cosas por medio del Derecho Civil, y cómo 
los pueden alterar y mudar para vivir con más comodidad y unión, cuyo establecimiento ¿̇cómo era posible que se lograra, ni que subsistiera en medio de tan diversas pasiones y genios, a no servir de norma las mismas máximas que cada uno tiene sembradas dentro de su propia razón? De aquí nace que se siente y percibe desde luego, que degenera y desvía en iniquidad y que puede corregirlo y templarlo el que se ejercita en el estudio de sus fundamentales documentos. Por fin, éste es el único camino para hallar medios de decidir los negocios con los que no viven sujetos a unas mismas leyes y para determinar todos aquellos casos que, por más que se desvele la jurisprudencia, siempre quedan fuera de su alcance.

La utilidad y su necesidad, la evidencia y universalidad con que la mano poderosa de Dios la comunicó a los hombres, son el carácter más distinguido de esta ciencia. El género humano pudiera subsistir sin muchas de las artes y descubrimientos, aunque saludables, que el tiempo y la industria unidos han facilitado; pero no sin la inteligencia de la bondad y rectitud de las acciones, porque creeríamos que hubiesen imágenes del Sumo Hacedor sin participar de su virtud y sin relación a sus atributos, que es un grande absurdo del entendimiento. Así, no nos debemos persuadir que estuviese apagada en los siglos que precedieron esta admirable antorcha, sino que alumbró con igual esplendor a los hombres en todos tiempos, bien que, a medida que la malicia y la ignorancia cubrían de tinieblas el mundo, fue más o menos escaso su aprovechamiento.

11

\section{Origen y antigüedad de este Derecho}

Sabemos, no obstante, que algunos historiadores han declarado a Grocio por el primer autor y escritor de este Derecho, pero esta proposición sólo se debe admitir en cierta parte, en que es verdadera. En primer lugar, no puedo creer que cuando Grocio dijo en sus doctos prolegómenos ${ }^{3}$ Veterum Philosophorum nihil exstat hujus generis, fue para hacerse inventor o descubridor de las máximas con que la razón se conduce, como quiere dar a entender Christoval

3 Grotius, De Jure belli ac pacis, Proleg. § 36. 
Augusto Heumano4. Tenía Grocio sobrados méritos sólidos para no arrogarse esta postiza gloria, y los tuvo también para triunfar de cuantos después le han combatido valiéndose de su propia doctrina. Además, que él mismo manifiesta el modo con que se aprovechó de los filósofos antiguos y, así ${ }^{5}$, esta proposición suya se debe regular por otras que tenía vertidas en los mismos prolegómenos, los cuales empiezan diciendo: Jus civile, sive Romanum, sive quod cuique patrium est, aut illustrare commentariis, aut contractum ob oculos ponere, agressi sunt multi, at jus illud, quod inter populos plures, aut Populorum Rectores intercedit, sive ab ipsa natura profectum, aut divinis constitutum legibus, sive moribus, aut pacto tacito introductum, attigerunt pauci; universim, ac certo ordine tractavit hactenus nemo, cum tamen id fieri intersit humani generis. Y después, habiendo descubierto el designio que llevaba de tratar de la guerra y de las causas que la pueden ocasionar entre las naciones, y reglar los medios de sus diferencia por los principios universales de justicia, añade ${ }^{6}$, poco antes de la proposición que se le increpa: Eo autem majus visum est pretium operae, quod, uti dixi, totum hoc argumentum tractavit nemo, et qui tractarunt partes ita tractarunt, ut multum relinquerint alienae, industriae. Y en este sentido es del todo cierta su proposición porque no había ninguno que con la competente generalidad, ni con el método debido, hubiese manejado este argumento, extendiéndolo a todo cuanto puede abrazar y penetrando hasta las mismas fuentes de toda la justicia para reducirlo a reglas ciertas. Esto es lo que hizo Grocio, pero confesando con candor e ingenuidad una gran deuda a los que le habían precedido, pues descubre, sin rebozo, en los citados prolegómenos, el método de que se valió para recoger las máximas de los libros sagrados, de los sabios antiguos, filósofos, jurisconsultos y teólogos morales. Y cuando no lo confesase, están sus márgenes y notas por lo común ocupadas con sus autoridades, entre cuyo número sobresalen las de Santo Tomás, con las de Victoria, Soto, Medina, Ayala, Covarrubias, Menchaca y otros sabios españoles.

12

[Primera] Formación de esta ciencia

Amaneció muy temprana la luz que muestra a los hombres el camino de su

4 Heumann, Conspectus reipublicae literariae, cap. 5 .

5 Grotius, De jure belli ac pacis, Proleg. $\S 42$.

6 Grotius, ibid., § 36 . 
propia perfección, y, por el tanto, debemos convenir en que esta materia ha sido conocida por todos; para cuya comprobación, sin duda, sirve mucho la curiosa investigación de aquellos historiadores que se empeñaron en descubrir las primeras memorias de esta ciencia. Estos tales hallaron sin mucha dificultad continuos vestigios y copia de testimonios desde la antigüedad más remota, por cuyo trabajo merecen las más justas alabanzas. Pero no son tan acreedores cuando, por no contentarse con este triunfo, y dejándose deslumbrar de su propio entusiasmo, llegaron a figurarse que muchos de los filósofos habían sido versados y ocuparon sus meditaciones en este determinado estudio, citando, por ejemplo, los estoicos, los platónicos, lo cual mismo hicieron entre los jurisconsultos, y escritores eclesiásticos y teólogos, formando épocas y clases por la serie y progresión de sus diversos conocimientos. Esta fue una ilusión de su fantasía porque lo más que se puede decir es que no ignoraron los antiguos las principales máximas y documentos como tan necesarios, y que hicieron en muchas ocasiones la aplicación y uso con puntualidad y destreza. Mas los principios solos no comunican toda la virtud que el entendimiento necesita para su ejercicio. Los principios adquieren segunda fuerza, y llegan a tener el complemento de toda su perfección, cuando, unidos la meditación y el ingenio a fuerza de combinaciones, hallan la relación que tienen entre sí y con los de otras ciencias cuando después de repetidas operaciones y pruebas se va descubriendo el ámbito y globo de materias donde pueden alcanzar todas sus líneas, y, por fin, se halla el arte y preciosa economía de aplicarlos a las especies y casos que ocurren. Este género de Filosofía quedó reservado hasta principios del siglo pasado, en que se echaron los primeros cimientos y, prosiguiéndose después por todo él, se fueron reuniendo todas las máximas y preceptos del Derecho Natural y de Gentes, separándolas por sus clases y formando un cuerpo de doctrina. Así se halló descubierta una nueva ciencia y arte, que fuera el más glorioso monumento de cuantos ilustran al humano espíritu si los que se dedicaron a su investigación hubieran siempre navegado por alta mar, sin tocar en las costas, que están ocupadas, todas, de las humanas pasiones. $\mathrm{Y}$ entre todos cuantos pueden competir al triunfo de este descubrimiento, sin duda ninguna que se puede contar por el principal a Grocio, pues, aunque el título de su obra parece particular y limitado, dejó en el fondo erigida una copiosa biblioteca que ha enriquecido a cuantos después la han frecuentado. Y así, siendo ya tiempo que hablemos de los progresos de esta ciencia, parece justo que el catálogo de los autores empiece por su nombre. 
13

\section{Progresos y Escritores principales}

Hugo Grocio

Hugo Grocio nació en Delf, pueblo de Holanda, por los años de 1583. De tan temprano y sublime talento que, siendo aún sólo de nueve años, poseía la Poética y hacía versos con perfección; a los quince tenía los grados de Filosofía y Jurisprudencia y estaba prodigiosamente instruido, no sólo en ellas, sino en la Astrología, Matemáticas y en la Teología. Luego se entregó al foro y contribuyó a dar a conocer su elocuencia y vasta erudición, y a que lo nombrase por su síndico la ciudad de Rotterdam. En este empleo se mantuvo hasta que, encendiéndose dos partidos contrarios de los gomaristas y ariminianos, cada uno de los cuales se esforzaba para que prevaleciesen las opiniones de su secta, sospechando que favorecía a los ariminianos, fue Grocio perseguido por el bando contrario y puesto en la cárcel, con riesgo de perder la vida, a no haberlo sacado de ella con ingenioso ardid su mujer, María Reigersberg, metiéndole dentro de un cofre con que solían llevarle libros. Estando ausente de su patria halló generoso abrigo en la Francia, para cuya corte, habiéndosele dado después la embajada de Suecia, emprendió allí, a ruegos y con el favor de Nicolás Peiresk, gran protector de todos los hombres sabios de su tiempo, que hizo se le encargase también por el rey de Francia, la excelente obra que concluyó y publicó, dedicada al mismo rey Luis XIII, en 1625, con el título De Jure Belli et Pacis. Escribió otras muchas obras, ilustrando varios autores de la antigüedad, la Historia de su país, que pasa por modelo en este género; trabajó sobre la Escritura, y dio otros muchos tratados, estimables por su gran erudición y profunda doctrina. Pero, entre todos ellos, sobresale esta obra, como él mismo lo juzgaba así en sus cartas, a causa de que, según refiere Paulo Colomies 7 , apuró en ella Grocio todas las fuerzas de su talento. Tuvo pensamiento de intitularla del Derecho Natural y de Gentes, como lo es en sustancia, y la llamaba así hablando con sus amigos ${ }^{8}$. Pero mudó de dictamen y le puso el título de Jure Belli et Pacis para que, atraídos y cebados con el frontispicio, los que gobiernan las armas y los que están al lado de los príncipes lo leyesen y se aprovechasen de su lectura, temiendo que

7 Colomiès, Bibliotheque choisie.

8 Véase la vida de Nicolas-Claude Fabri de Peiresc escrita por Pierre Gassendi el año 1623 . 
de otro modo lo desecharían, por contemplarlo como una obra de escuela y pura curiosidad. Así lo refiere él mismo en su carta, que publicó Paulo Colomies $^{9}$. Y, a la verdad, no le engañó su deseo, porque ya en 5 de marzo de 1632 le escribía Monsieur Bignon, su amigo y sabio magistrado de Francia, cómo había entendido que Gustavo Adolfo de Suecia nunca lo dejaba de vista ${ }^{10}$. De manera que la obra de Grocio, en breve, se fue haciendo el libro manual de los príncipes y todos los hombres de Estado, y quedó adoptado y leído en muchas universidades de Alemania. Murió Grocio en Rostock, ciudad del Mekelburg, estando de vuelta de Stokolmo, en 1645, a los sesenta y dos años de edad, de donde lo trasladaron a Delf, lugar de su nacimiento.

Dos cosas prueban el gran mérito de este tratado: la una, que no hay obra que haya sido más impugnada por los que después escribieron, sin embargo de haberla disfrutado bien, porque son tan excelentes sus meditaciones y tal la rectitud de su juicio, que, no pudiendo negarle la preferencia, no quedó otro partido a la emulación que el de oprimirlo; la otra, que no hay autor clásico, exceptuando los de la antigüedad, que haya merecido mayor número de comentarios. Viendo lo cual, decía ya Puffendorf, que, según la prisa que se daban, era de recelar que con el tiempo tuviese Grocio tantos ilustradores como Pedro Lombardo o las Institutas de Justiniano ${ }^{11}$. Entre todos ellos, los más estimados son Juan Friderico Gronovio, que ocupa un lugar muy distinguido en esta especie de literatura, y le puso notas escritas y filológicas muy breves, y que conducen para su mayor inteligencia, aunque no sintió tanto de ellas Juan Barbeyrac ${ }^{12}$. Estas notas de Gronovio se adulteraron y aumentaron por otra mano, a nombre suyo, y se ha de preferir la edición de Ámsterdam de 1650, en sentir de Colomies ${ }^{13}$. Enrico Boeclero retocó también esta obra con mucho juicio y con igual doctrina, en juicio de Puffendorf, esparciendo gran copia de testimonios y ejemplos de la antigüedad. Otros comentadores la examinaron sobre los principios del Derecho Natural, ya confirmándola, ya contradiciéndola, como Gaspar Zieglero en Witemberg 1666, Enrique Heninges

\footnotetext{
9 Epistolae clarorum virorum, Epist. 3.

10 Colomiès, Bibliotheque choisie.

11 Pufendorf, Specimen controversiarum, cap. 1.

12 Barbeyrac, Prefatio in Grotium.

13 Colomiès, Bibliotheque choisie.
} 
en 1673, y el mismo Juan Barbeyrac que, sin embargo de haberla traducido primero en francés Monsieur Courtin, la volvió a traducir y le puso notas, tanto al original como a su traducción, de dos especies: las unas, críticas, explicando el contexto y restituyendo sus citas viciadas por el cotejo que hizo con las fuentes; las otras, doctrinales y de su Jurisprudencia Natural. Por último salió a luz el Comentario del barón Enrique de Coccei que añadió después, en edición posterior, su hijo el barón Samuel de Cocoei, y es el más completo y apreciado por todos. Se compone de cinco tomos en cuarto, impresos en Lausana, en los años 1751 y 1752, de los cuales: el primero es una introducción a Grocio que hizo Samuel de Coccei por medio de doce excelentes disertaciones sobre los principales puntos de su doctrina, y en la última presenta un nuevo sistema del Derecho Natural y Romano, hermanando los dos o reduciendo la jurisprudencia de los jurisconsultos romanos a sus primitivas fuentes; los cuatro últimos contienen una ilustración continuada sobre el texto de Grocio, que se pone entero, con las notas que el mismo Grocio añadió, las de Gronovio, Barbeyrac y, después siguen aparte las observaciones de los Cocceis, el padre e hijo, que es obra toda de mucho trabajo y erudición.

Con el mismo fin, aunque por diverso camino, trabajaron otros para hacer más perceptible la lectura de Grocio, considerándola llena de preciosa doctrina e inmensa erudición. Así, Jorge Kulpisio publicó el Collegium Grotianum, que sólo sirve para preparar a su estudio, explicando los lugares obscuros y ampliando los que son demasiado concisos. Juan Schefero lo redujo a compendio para que fuese más accesible a los jóvenes, intitulando su obra Grotius ennucleatus, compuesta para el uso del conde de la Gardie, y que celebra mucho Struvio ${ }^{14}$. En cuyo particular acaso aventajan a todos las Praelectiones in Grotium de Juan Gottlieb Heineccio, donde redujo a breves tesis toda su doctrina y presentó con la mayor claridad la fuerza de sus argumentos, cuyas circunstancias hacen sea el mejor maestro y guía que pueden tomar los aficionados, para que no les embarace lo elevado y copioso de la materia.

Grocio se distingue por varias opiniones singulares que han sido ya refutadas,

14 Struvius, Bibliotheca juris selecta, cap. 18. 
ya aprobadas, por los que le han seguido; como la del consentimiento y modo de opinar uniforme de los hombres, que toma por argumento del Derecho Natural y de Gentes; el Derecho Positivo y voluntario, que estableció entre ellas; la doctrina de los Reinos Patrimoniales y Usufructuarios; el Derecho para castigar e imponer penas entre iguales, que señaló por una de las causas justas de la guerra, sin otras menos considerables. Conserva también, por otra parte, muchas ideas y nociones de su propia invención; como los títulos de adquirir las cosas originarios y derivativos, el modo de ocuparlas per fundos o per universitatem, la distinción de dominio eminente y vulgar, etc. Me he detenido algo más en Grocio porque es el autor más principal en esta materia, pero sin canonizar toda su doctrina, de la que se advertirá lo conveniente, como de todas las demás.

17

\section{Juan Seldeno}

Contemporáneo de Grocio fue Juan Seldeno, hombre docto y de muy profunda erudición, que nació en Salvintg, de la provincia de Subtsex, en Inglaterra, el año 1584, y murió en 1654. En su obra de Jure Naturali et Gentium secundum doctrinam Hebreorum quiso probar que Dios dio a Adán los principales preceptos de este derecho, reducidos a siete, después a Noé, y de éste vinieron conservados por la tradición a todos sus descendientes. Pero la antigüedad y verdad de esta tradición está muy dudosa y débil, sobre ser muy imperfecta y reducida esta doctrina para comprender al Derecho Natural.

18

\section{Thomas Hobbes}

No muchos años después dio la Inglaterra otro ingenio de una viveza y penetración singular, pero de mayor osadía y libertad. Thomas Hobbes, que es de quien hablamos, tuvo su nacimiento en Malmensburi, en 5 de abril de 1588, y vivió hasta el año 1679, en que murió a los noventa y un años de edad. Escribió la obra de Cive, que es la concerniente a nuestro asunto y que no vio la luz pública hasta el año 1646, donde redujo a 20 todas las leyes naturales. Figurose los Derechos de los hombres por sus deseos y, contemplándolos como suelen ser, sentó que el estado natural es hostil y de una guerra de todos contra todos, bellum omnium in omnes, donde, por consecuencia, todos tienen 
derecho a todo, jus omnium in omnia ${ }^{15}$. Fue el primero que pensó en deducir de sólo un principio las reglas del Derecho Natural ${ }^{16}$; el que introdujo el método geométrico y matemático ${ }^{17}$; y el que reunió y confundió el Derecho Natural con el de Gentes, diferenciándolos sólo en el objeto, porque el uno dirige los hombres particulares, y el otro las Gentes y Naciones ${ }^{18}$. Este mismo, meditando sobre la construcción y forma de las Sociedades Públicas o Estados, cimentó la doctrina de los pactos y decretos que intervienen, cuyo plan han adoptado después otros modernos, y tiene, además, otras ideas y opiniones muy particulares, pero éstas forman la divisa y como seña de su doctrina. Ha sido refutado por muchos, pero ninguno hizo ver más clara la falsedad de sus principios que Ricardo Cumberland, inglés también, en su ingeniosa y erudita obra Tractatus Philosophicus de Legibus Naturalibus, traducida al francés por Juan Barbeyrac.

\section{Samuel Puffendorf}

Iba tomando cada día más aumento esta ciencia, que empezaron, por lo mismo, a llamarla Jurisprudencia Universal, y remontó a lo más alto su vuelo con las obras de Samuel de Puffendorf. Su patria fue Hoeha, lugar de la Misnia, en el electorado de Sajonia, el 8 de enero de 1632; murió por los años de 1694. Este, aprovechándose de la lectura de Grocio y Hobbes, compuso primero una obra con el título Elementa Jurisprudentiae universalis, que dedicó a Carlos Luis, elector palatino, ciegamente apasionado por Hobbes, en remuneración de lo cual erigió una cátedra en Heidelberga del Derecho Natural y de Gentes, que fue la primera de este estudio, y se la dio a Puffendorf. Después, siendo llamado por el rey Carlos XI de Suecia para enseñar el Derecho, publicó su obra de Jure Naturae et Gentium, en 1672, cuya edición es la más correcta; pero la aumentó de una cuarta parte en 1684, y contrajo la propia doctrina en otra más pequeña, de Officio Hominis et Civis. La principal de todas es la de Jure Naturae et Gentium, donde se introdujo a buscar los principios de las acciones humanas antes de tocar las materias de derecho y, tratadas éstas, pasó a indagar el origen y naturaleza de los Estados, con lo que empezó a in-

\footnotetext{
15 Hobbes, De cive, Epist. Dedic. et Praefat.

16 Hobbes, Epist. Dedic.

17 Hobbes, Epist. Dedic.

18 Hobbes, De cive, cap. 14, § 4.
} 
corporar en este estudio lo más acendrado de la Moral, de la Jurisprudencia y de la Política, y vino a hacer más familiar la Filosofía de los Oficios. Casi todos los demás modernos han adoptado este propio rumbo, por cuya causa está reputado por el primero que formó un sistema y cuerpo formal o regular de esta materia, que es lo que él mismo dice que se propuso ${ }^{19}$. Juan Barbeyrac tradujo al francés su Derecho Natural y de Gentes, e hizo todavía más, porque lo pulió corrigiendo sus citas falsas, sus inconsecuencias y oscuridades, y lo ilustró, por último, con notas; de modo que su traducción se estimaba ya más que el original en tiempo de Burcardo Struvio ${ }^{20}$. Pero Puffendorf, sin embargo, que tenía una lectura vasta, y dio a conocer más la extensión de este argumento, tropezó en errores muy crasos. Descubre lo bastante su adhesión, aunque disimulada, a Thomas Hobbes, su celosa emulación a Grocio, y un empeño formado de impugnar y contradecir a todos lo que debilita en gran parte su dictamen. Dejo por bien conocida, como despreciada de todos, la Teoría o laberinto de sus entes morales con que principia, y que sólo sirve de confusión, con otros defectos que, por comunes a muchos, se apuntarán en general. Nicolás Hercio le puso notas en 1706, y Gottfrido Mascovio lo publicó en dos tomos en cuarto, en 1744, con las notas enteras de Hercio y extractando lo principal de las que Barbeyrac dio a su versión francesa. Juan Bautista Almici lo reformó de sus proposiciones erróneas y lo imprimió poco ha con propias ilustraciones.

20

\section{Christiano Thomasio}

Quedaba por fin (dicen los alemanes protestantes) reservado el poner la última perfección a ésta obra para Christiano Thomasio, natural de Leipsik, en Sajonia, donde nació el año 1655, y murió en 1728. Dos son, en especial, sus obras sobre este asunto: la una, Institutionum Jurisprudentiae Divinae libri tres, in quibus fundamenta Juris Naturalis secundum hypotheses illustris Puffendorfi perspicue demonstrantur; y la otra, que es la más principal, Fundamenta Juris Naturae et Gentium ex sensu communi deducta, in quibus ubique secernuntur principia honesti, justi et decori. Fue su ánimo distinguir lo justo de lo honesto y decoroso, tan estrechamente unidos que no es fácil separarlos sino por la mayor o menor fuerza con que se prescriben unas

19 Pufendorf, Specimen controversiarum, cap. 4, § 11.

20 Struvius, Introductio in notitiam rei litterariae, cap. 5 . 
obligaciones respecto de otras. Pero todas están dictadas por la ley natural y se dirigen a un justo fin; no dimanan de la utilidad, comodidad o precisa indigencia nuestra, como puros instrumentos que sirven para captar el agrado de los otros. Si así lo creyeron, como se les arguye, Christiano Thomasio y sus secuaces, atribuyendo lo justo sólo al Derecho Natural y separando lo que es honesto y decoroso, cortaron con fatal golpe tres ramas entrañablemente unidas de un mismo tronco. De aquí ha procedido aquella preliminar distinción de los oficios de justicia y humanidad, tan común entre los modernos, que usada para solamente aclarar ciertas especies y materias, reduciéndolas a principios del Derecho Natural con su género de obligación, podrá ser útil; pero, si no se procede con la necesaria moderación, padecerá los mismos inconvenientes.

\section{Juan Gottlieb Heineccio}

Juan Gottlieb Heineccio vio la primera luz en Eisemberg, lugar principal de Altemburg, en la Thuringia, provincia del electorado de Sajonia, el año de 1681; y después de una lustrosa carrera literaria, en que fue profesor de varias ciencias y en distintas universidades, murió en 1741. Su vida laboriosa y decidida pasión por todas las materias del buen gusto hicieron que se aprovechase de los trabajos de todos con fruto muy conocido, como lo publica su libro Elementa Juris Naturae et Gentium, que dio a luz en 1737. Este es un breve compendio, muy agradable y sazonado (como que lo escribió en los últimos períodos de su edad), donde se ven floreadas y recogidas todas las opiniones y más particulares meditaciones de cuantos le habían precedido: de Grocio, Hobbes, Puffendorf, Thomasio, combinándolos a veces y dando voto sobre ellas, pero con hermosura de estilo, con exquisito gusto, con perspicuidad, método y amena erudición, que todo le era familiar.

22

\section{Christiano Wolfio}

Después de Heineccio escribió Christiano Wolfio, este filósofo y matemático tan conocido que dio Breslau, capital de la Silesia, en 1679, habiendo vivido hasta el año 1754. Desde sus primeros años tuvo formada resolución de tratar este aspecto, que hacía días era uno de los objetos más deliciosos entre los eruditos. Corrió antes por el vasto campo de las ciencias, en que consiguió 
mucho nombre y celebridad por Europa, y después dedicó todas las fuerzas que iba reservando su talento para escribir su Derecho Natural y de Gentes, que publicó en nueve tomos en cuarto después del año 1740, disponiéndolo como tan versado que era en el método matemático. Esta última circunstancia no le favoreció tanto como pensó Wolfio, porque hace embarazosa y molesta su doctrina la continua y necesaria remisión a otras obras suyas, y los rodeos de que se vale aun en las cosas más claras para proponerlas en sus acostumbradas demostraciones. Con todo, nadie le puede disputar la gloria de haber sido el que puso la última mano, y el que completó y redujo a perfecto orden y sistema al Derecho de Gentes. Hasta su tiempo apenas se distinguía del Derecho Natural, y los más se habían dado por satisfechos con establecer los preceptos naturales, haciéndolos comunes a los Estados y a los individuos. El barón de Volf, cuyo crédito le había granjeado esta nueva dignidad, tomó pues por empeño tratar aparte cada cosa, y dar a conocer su peculiar conducta y naturaleza. Porque los Estados, aunque los unos respecto de los otros se puedan considerar como otras tantas personas particulares que viven en el estado natural, y, por lo mismo, les correspondan los mismos derechos y obligaciones que a los particulares; los Estados, además, contemplándose como personas morales que resultan de la asociación y formación de los cuerpos políticos, se distinguen necesariamente, y por muchos respectos, de los individuos físicos. Y siendo distinta su esencia y naturaleza, los oficios y derechos que les corresponden, que siempre son y deben ser los que pueden adaptar a la naturaleza de los sujetos, han de ser muy diversos, o su aplicación se ha de diferenciar por la distinta índole de la persona. Sobre este principio va recorriendo por todos los oficios, derechos y acciones que son propios de las gentes y sociedades con mucha invención y fertilidad. Esta obra se reimprimió posteriormente en el año 1764, en Venecia, con las observaciones de Watel, también en nueve tomos.

Sobre los mismos principios y plan de Wolfio, perfeccionó últimamente el Derecho de Gentes Monsieur de Watel, suizo, y lo publicó en francés el año 1758, con el título Droit des gens ou principes de la Loi Naturelle appliqués á la conduite et aux affaires des Nations et des Souverains par Monsieur de Watel; en donde, suavizada la sequedad y aspereza del método de Wolfio, no sólo 
está amenizada la materia, sino que, por el buen orden y copia de ejemplares modernos con que lo ilustra, tiene mucha mayor claridad, de modo que, hasta el día, es la obra mejor que ha salido del Derecho de Gentes.

\section{4 \\ Monsieur Burlamaqui \\ Felice}

Monsieur Burlamaqui, profesor de Ginebra, mereció también en estos años pasados el aprecio y voto de muchos en la obra que escribió con el título Principes du Droit Naturel, Principes du droit Politique, por la precisión, buen orden, método y claridad; con la cual creían poder instruirse en esta ciencia sin necesidad de acudir a Grocio y Puffendorf. Pues, aunque los dos están reputados por los autores más clásicos de ella, sus obras no se pueden considerar por elementales: la de Grocio, porque la profundidad de su doctrina, tratada muchas veces con demasiada brevedad, pide lectores instruidos y que piensen continuamente sobre lo que leen; la de Puffendorf, con efecto contrario, por la mucha prolijidad y confusión. Por estas causas volvió a publicar Monsieur Felice, otro profesor de Ginebra, la obra de Burlamaqui, mudando a la segunda parte el nombre de Derecho Político en Derecho de Gentes, que le correspondía con más propiedad, y haciendo otras mutaciones en algunos capítulos, pero aumentándola toda con nuevas notas y observaciones suyas, la que imprimió en Yverdon en 1766, en ocho tomos en octavo, con el título Principes du droit de la Nature et des Gens. Después, redujo toda esta doctrina en cuatro tomitos más pequeños, impresos también en Yverdon, en 1769, con el título Leçons du droit de la Nature et des Gens, para que sirviese de breviario o manual introducción a los que quisiesen emprender esta carrera.

\section{5}

\section{De otros Escritores en común}

Más se debe advertir que, en la serie historial de los autores, sólo se han puesto los que con razón se pueden llamar más clásicos y principales, ya sea porque con la feliz invención de sus sublimes ingenios fueron los que primero formaron el cuerpo de esta ciencia, ya porque con el fondo de doctrina, o por su excelente método, le dieron elevación y dignidad. Restan otros que, aunque no ocupen el primer grado, pueden aliviar mucho el trabajo a los que 
desean instruirse en los principios de este estudio. Tales son Felipe Reinhardo Vitriario: Instit. Jur. Nat. et Gent. ad method. Hugonis Grotii; el padre Ignacio Schwarz: Instit. Jur. Nat. et Gent.; el padre Anselmo Desing: Larva detracta et Jus Nat. et Gent.; Lorenzo Fleischero: Instit. Jur. Nat. et Gent.; J. Justino Schierchmidio: Elem. Jur. Nat. et Gent., Joaquín Jorge Daries: Instit. Jurisp. univer.; Juan Jacobo Schmausio: Nuevo sistema del Derecho Natural, que publicó en lengua alemana, en Gottinga el año 1754, y es adoptado y seguido últimamente por los alemanes; Tadeo Werenko: Jus Nat. et Gent., impreso en Venecia en 1767; Juan Bautista Almici: Instit. Jur. Nat. et Gent., en Brixia, año 1768; sin otros de cuyas obras, por servir para la inteligencia de algunos de los escritores arriba referidos, se hizo mención en cada uno de ellos, como de los Cocceis en Grocio, de Cumberland en Hobbes, de Barbeyrac en Puffendorf, etc.

\section{6}

\section{Montesquieu}

Con esta propia mira no será de extrañar se haga memoria de ciertas obras más modernas, cuyos autores, aunque no han tomado por asunto todo el derecho en general, se han señalado por algunos especiales descubrimientos y nuevos rumbos que han querido tentar. Es bien conocido de todos el barón de Montesquieu, Carlos de Secondat, que nació en el castillo de la Brede, cerca de Burdeos, en 1689, y murió en París, en 1755. Su obra, publicada en francés, L'esprit des lois, ha corrido y corre, ya vertida, ya en su idioma propio, con mucho aplauso por toda la Europa, en la que, además de que se excitan muchas observaciones profundas sobre la esclavitud, sobre el derecho de la guerra y conquista, y en otros varios puntos de esta materia, es muy notable su nuevo modo de pensar, por lo menos en lo que toca a la naturaleza y forma de los Estados. Porque, apartándose del camino que hasta su tiempo habían seguido todos los políticos, no los divide en monárquico, aristocrático y democrático, ni se ocupa en indagar los mixtos e irregulares, sino que sólo conoce tres especies, pero muy distintas entre sí, a saber: el republicano, el monárquico y despótico. En estos mismos, a fuerza de combinaciones políticas, ha encontrado la singular diferencia que media entre la naturaleza y organización de un Estado con su principio o causa que le da impulso para que obre. Sobre cuyos fundamentos ha levantado otro nuevo plan, que, además de servir como de balanza en que se puede pesar la felicidad de cada Estado, 
es necesario conocer para entender muchos autores modernos, porque casi todos se revisten de su mismo espíritu.

Linguet

Contra éste, contra Puffendorf y Grocio, y con menosprecio de los autores del Derecho, pero sobre todo contra Monsieur de Montesquieu, se dirige la obra anónima, en francés, con el título Théorie des lois Civiles, impreso en 1767, y que se dice ser de Monsieur Linguet, abogado de París. Obra concebida en un grande ardor de la imaginación, a fin de lisonjear a dos ídolos muy propios de ella, el despotismo y el amor de la propiedad, que se declaran por inventores de todas las leyes e institutos humanos.

28

Rousseau

Otros sistemas ha intentado establecer Juan Jaime Rousseau, ciudadano de Ginebra, tanto sobre los principios de la política, en su contrato social, escrito en francés con el título Du contrat social ou Principes du droit Politique, en Ámsterdam 1762; como sobre el Derecho Natural, en su discurso sobre la causa de la desigualdad entre los hombres, su título, Discours sur l'origine, et les fondements de l'inegalite parmi les hommes, en Ámsterdam 1755. Su extraordinario modo de pensar en estas materias, opuesto a todo el buen orden y la quietud pública, ha sido justamente despreciado y proscrito en todas partes, por cuya causa no es razón que me detenga más.

29

Escritos modernos Detestables

Aulo Gelio refiere ${ }^{21}$ que se acordó en Asia, por común resolución, que nadie se atreviese a proferir el nombre del que había quemado el templo de Diana. Este fue Herostrato, que juzgó dejaría de sí perpetua memoria aplicando las sacrílegas teas al famoso santuario de Efeso. Igual castigo merecen las obras de ciertos faccionarios modernos, que de común intento conspiran a destruir

21 Gellius, Noctes Atticae, lib. 2, cap. 6. 
la Legislación y la Moral. Con todo, la casualidad puede hacer que se encuentre con el Emilius, l'Esprit, Systeme de la Nature, y otros partos semejantes, y muy conformes todos en el propio designio y doctrina; en este caso es necesario recurrir, desde luego, a los escritos de Monsieur Bergier, teólogo y canónigo de la Iglesia de París, dotado de particular talento para curar estas enfermedades, y en especial a los dos libros publicados en París el año 1771, en dozavo, con el título Examen du Matérialisme ou refutation du Systeme de la Nature, donde plenamente convence y descubre sus falacias.

30

\section{Vicios y defectos de muchos modernos}

Ni se debe juzgar nunca que se aprueban los tratados de los demás autores referidos, aunque se nombraron con indiferencia, cuando poco antes, al hablar de Grocio y Puffendorf, ya quedó reservado para este lugar advertir lo que se debía llevar prevenido. Hay ciertos defectos comunes en que inciden todos los más de los modernos, y que es necesario tener conocidos para no caer en sus lazos. Por lo común, todos concurren a desarmar la autoridad, negando la veneración y asenso que se debe a los autores, tanto sagrados como profanos, sobre la suposición que no merecen más fe sus testimonios que en cuanto van conformes con la recta razón. Este es el juez y árbitro absoluto que sólo reconocen, porque cada uno le tiene ganado dentro de su casa, de suerte que persuaden a los demás a que no aprendan por el mismo camino que ellos aprendieron, que fue aprovechándose de los otros. Así fundado el tirano reino de la razón, ya no consultan, para derivar el Derecho Natural, a los Libros Sagrados; desprecian los Santos Padres, los teólogos, los escolásticos y jurisconsultos; fiados en una serie de raciocinios que cada cual se esmera en ordenar con más artificio. El principio de la obligación, y todos los derechos, lo colocan en los pactos y convenciones, desconociendo la moralidad, torpeza o rectitud intrínseca en las cosas, que les hace ser en sí buenas o malas, independiente de los humanos institutos. La ley más sagrada, que en su modo de pensar tienen intimada los hombres, es la de procurar su utilidad y conservación, y alejar lo nocivo y dañoso, máxima fundamental sellada a la frente de sus escritos, con la que infunden sólo amor a lo sensual y terreno, como los epicúreos, sin levantar los ojos, para no acordarse de su más elevado destino. Y a este tenor se va eslabonando una cadena de principios arbitrarios y propios sólo para engañarse a sí, porque sueltan con toda libertad las riendas a 
su discurso, mientras tienen cautivo el corazón de sus particulares pasiones. De donde nace no reputar al matrimonio sino como una pura especie de contrato; a la Iglesia, como una sociedad menor, o colegio, al modo de uno de los gremios inferiores; con otras proposiciones dignas de severa censura.

\section{Modo para conocer los Autores sospechosos}

Son sabidos los lugares donde suelen, por lo común, verter las máximas perniciosas, y así, para descubrir desde luego en cualquiera autor que se tome en las manos su buena o mala creencia, se debe ver, sobre lo que queda advertido en el número antecedente, cómo trata de los principios de las acciones humanas y de la diferencia entre ellas, porque coartan estos tales el libre albedrío del hombre, y las suponen buenas o malas a medida sólo que las ven aprobadas o prohibidas por los legisladores civiles, negándoles la moralidad intrínseca; la obligación hacen que derive sólo del impulso interior y aprobación del juicio, quitándole su principal fuerza que consiste en el precepto de la voluntad divina; disminuyen el valor del juramento, porque separan el vínculo de la religión; ponen varias clases con sus peculiares nombres en la verdad y mentira, para usar con más ensanche; juzgan por precisa la sociedad conyugal, y en ciertos casos por disoluble; la formación y origen de los Estados, según muchos de ellos, fue torpe e injusto para un bien de pura conveniencia, que es lo que sólo se proponen, prescindiendo de la justicia y religión; otros no hallan en la suma potestad sino un encargo y administración amovible a voluntad del pueblo, en quien se figuran que está radicada la soberanía; y casi todos cuentan por uno de los derechos de la majestad el poder absoluto sobre los ministros y cosas sagradas, y sujetan la religión y el culto al arbitrio del Gobierno. No acuerdo sino parte de sus comunes vicios, porque sería sobrado prolija la enumeración de todos ellos, y bastan estos avisos para que leyéndose un autor se conozca si es o no sana su doctrina; pues cuando halle que sostiene alguna de estas proposiciones, seguramente lo puede desechar por sospechoso.

32

Autores Católicos con que se refutan

Si después de conocer las faltas de los autores se desea además saber el modo 
como se refutan, para estar prevenidos y más fortalecidos en la verdadera opinión, será menester recurrir a la lectura de nuestros apologistas, como el padre Ignacio Schwarz, en las Instituciones citadas; el padre Casto Ansaldo, De Principiorum Legis Naturalis Traditione; el padre Daniel Concina, que destinó todo el tomo sexto de su Teología para refutar todos sus errores; como lo hace por medio de varias disertaciones, con singular solidez y nervio el padre Anselmo Desing, en su Larva detracta, donde, al mismo tiempo que los impugna, trae mucha historia del origen y progreso de estas novedades, y añade las proposiciones que están ya condenadas por la Iglesia; y, por último, Juan Bautista Fineti, en sus doce libros De Principiis Juris Naturae et Gentium, impresos en dos tomos, en Venecia el año 1764, en los cuales, sobre los que tenían dicho los demás, añade nuevos argumentos y consideraciones con copia y elección de doctrina. Es necesario taparse algún tanto los oídos antes de entrar a escuchar las voces de algunos escritores, porque si no se aventura a quedar pervertidos con el delicioso encanto de sus pensamientos. Con esta mira se ha hecho la última edición de Heineccio en esta corte, añadiéndole las advertencias que han parecido más oportunas de los autores católicos, pues servirán de ejemplo para conocer el veneno de las proposiciones, y al mismo tiempo suministran los medios más eficaces para convencerlas.

\section{Precaución para establecer las reglas del Derecho Natural}

No dice con la piedad y moderación cristiana, el que se establezcan leyes o máximas en este Derecho sin que se sepa antes si son conformes con la doctrina revelada y tengan la censura teológica, en especial en lo que toca a las costumbres y modo de vida. Pues siendo Dios autor de las leyes divinas positivas y también de las naturales, las reglas y dictámenes de estas dos no pueden salir discordes. Y así, para asegurarse del todo en cualquiera ocasión que se ofrezca, convendrá consultar a los autores católicos que han tratado de propósito muchas de las materias del Derecho Natural y de Gentes, purificándolas de todos sus vicios y señalando la opinión que se debe seguir. Como Juan Sigismundo Stapff, Jus Nat. et Gen., impreso en Moguncia, en 1735, que contiene dos partes: el Derecho Público universal y el Grocio explicado. Tal es el padre Anselmo Desing, que a continuación de su Larva detracta pone otras dos obras, la una, con el título Jus Naturae liberatum ac expurgatum; la otra, Jus Gentium reductum ad limites suos; tal es el Taddeo Werenko, 
profesor del Derecho Natural y de Gentes en Dillinga, ciudad de Alemania, Jus Naturae et Gentium, impreso en Venecia, en 1767, y Juan Bautista Almici, que habiendo publicado el Derecho Natural y de Gentes, de Puffendorf, reformado, dió a luz en Brixia, el año 1768, sus Institutiones Jur. Nat. et Gent. secundum catholica principia, donde en substancia se refunden los elementos de Heineccio, sirviéndose de sus mismos capítulos, párrafos y aun voces, y teje por lo común su contexto de lo que Heineccio divide en párrafos y escolios, pero omitiendo, donde le parece, la expresión o cláusula que no se le acomoda, o advirtiendo alguna mutación. Todas estas precauciones son necesarias para proceder con acierto en este estudio.

Falsa preocupación de buscar un primer principio

Si se ha de tener alguna libertad, ha de ser para sacudir el yugo que ha oprimido a los más de los autores, porque creyeron que era necesario buscar un primer principio o proposición, que llaman fundamental, para deducir de ella, como consecuencia, todas las conclusiones de sus sistemas. Tras este suspirado objeto se les vio afanados, tanto como pudieran los egipcios por su perdida deidad, hasta encontrarle y fijarle, y son muchas las obras trabajadas con meditación y desvelo para este descubrimiento. Parece, por lo dicho arriba, que Hobbes fue el primer autor de este pensamiento, cuya misma idea abrazó Puffendorf, bien que sobre la elección de la proposición fundamental tuvo agrias y muy reñidas disputas con el padre Schwarz, Valentino Alberti, Velthemio y otros, de que da noticia en su Specimen controversiarum, añadido en la edición de Mascovio, capítulo cuarto, De Propositione fundamentali Legis Naturalis. Sobre lo mismo escribió después Enrique Bodino, Disert. de Jure Mundi, en Rinthel 169o. El barón Samuel de Coccei compuso varias disertaciones, que tomó por guía Heineccio en sus elementos, aunque muy justamente se duda si lo consiguió y desempeñó; Juan Shute, inglés, De Princip. Jur. Nat. et Gent.; Juan Bautista Vico, napolitano, De Jur. Nat. et Gent. principio uno et fine uno, en Nápoles 1720; el padre Nicolás Concina, dominicano, Origines, fundamenta et capita prima Jur. Nat. et Gent., en Padua 1734; Juan Dunio, napolitano, profesor del Derecho en Roma, disertación que publicó allí en 1760, en lengua italiana, con el título Saggio della Giurisprudencia unirversale, cuyos dos últimos sostienen la misma opinión de Vico; el padre Casto Inocente Ansaldo, De Principiorum Legis Naturalis Traditione 
libri tres, en Milán 1742; Carlos Pollini, abad de San Martín, De Juris Divini et Nat. origine, en Brixia 1750; Mr. Vollaston, inglés, Ébauche de la Réligion naturelle, sin otros a quienes inflamó el mismo supersticioso deseo.

Por ventura se libertaron de este fanatismo las demás ciencias, sin que no obstante hayan dejado de enseñarse con muy feliz progreso, pues ni la Teología, Medicina, ni Matemática, ni otra alguna, ha tenido precisión en sujetarse a un solo principio. Cuantos más y más claros se propongan, facilitan y fortalecen mejor las opiniones; pero si se hace alarde de no dejar el hilo que se tomó, este mismo empeño induce a interponer proposiciones y especies no necesarias, con sólo el fin de no cortar la tela como sucede a cada paso. Demás que nada podría saberse si no pudiese sosegar el entendimiento, hasta encontrar en cada punto la derivación del primer principio. Cuyas sólidas razones convencen la inutilidad de su sistema, como lo hace ver Juan Bautista Fineti $^{22}$, y han recabado, por fin, que se abandonase, pues nadie hace ya caso de este escrupuloso delirio.

36

Métodos que se proponen

A este espíritu de libertad, que es la que sólo conviene, se debe el haberse roto la cadena que estos mismos autores iban eslabonando con su reino de la razón; porque despreciaban en obsequio suyo a todos los maestros y escribían de nuestra Jurisprudencia con el mismo privilegio que M. Voltaire de la Historia, sin más apoyo que su discurso. La mayor parte de sus invectivas y censuras se dirige contra los escolásticos y sus métodos, como poco a propósito para el designio que meditan; y, en su lugar, admiten sólo tres por más oportunos y propios para esta enseñanza. El primero es el método axiomático, atribuido a Heineccio, porque es el que prefirió en sus obras y el que declara en su prefacio por más familiar a sus musas. En éste se procede por distinción y por axiomas o sentencias claras, que se van ordenando una tras otra hasta llegar a sus particulares conclusiones. El segundo es el demostrativo, llamado también científico, del que ya se valió Hobbes, y lo anunció en su prefación ${ }^{23}$;

22 Giovanni Francesco (Bonifazio) Finetti, De principiis juris naturae, lib. 7, cap. 7.

23 Hobbes, De cive, Praef. 
aunque otros juzgan que lo usó primero, ya entrado este siglo, Juan Ulrico, barón de Cramer. Su artificio consiste en formar una serie de raciocinios con mutua conexión para sacar las verdades que buscan. Y el tercero es el método matemático, bien conocido y común en las ciencias de esta naturaleza, donde se camina por supuestos y definiciones sin usar de término ni proposición que no esté antes confesada o convencida, y remitiéndose a las anteriores con que se enlazan para no dejar libertad al entendimiento. Este es el que Christiano Wolfio, como tan versado en las ciencias matemáticas, quiso introducir en el Derecho Natural y de Gentes con preferencia a los demás; pero juzgan lo contrario otros, hasta haberse encendido como una especie de guerra metódica, que así la llaman, según refiere Daniel Nettelblat ${ }^{24}$.

\section{7}

\section{No son necesarios}

Fuera muy loable decidir esta competencia cuando hubiesen probado que alguno de ellos es de toda necesidad preciso para el estudio de este Derecho. Están muy lejos de poderlo hacer, y sobre todo por lo que toca al matemático, que sólo llaman científico algunos, porque es el más incómodo e intempestivo, ya por la sequedad de haber de arreglar palabras y sentencias mondas llamándose entre sí recíprocamente, ya por la fatiga de llevar siempre preparada la red artificiosa; pues desde luego que falte un poco la ayuda de la memoria, desaparece de repente todo el espectáculo. La certidumbre de cualquiera ciencia consiste en la natural conexión de sus verdades con sólidos y claros principios, y hágase como se hiciere siempre resulta positiva evidencia; como la consiguieron todas las demás facultades, sin mendigar ninguno de estos métodos por tantos siglos que se han cultivado con amenidad y provecho.

38

\section{Del método Escolástico}

Ni se puede negar, sino con mucha injusticia, que el método Escolástico no sea capaz de conseguir esta gloria, tanto sino más que los que pretenden prevalezcan. El método Escolástico es el mismo que por algunos autores se llama Magistral, principia, por lo regular, previniendo lo que conviene separar del

24 Nettelbladt, Initia historiae litterariae juridicae universalis, § 288. 
objeto para no confundirle; puesto ya por este medio en claro, lo explican y apoyan con argumentos y silogismos, cuyo uso, si es moderado, es el más propicio para la convicción; y, por último, se hacen cargo y responden a cuanto les pueda impugnar. Así lidian campo a campo con el entendimiento, y no quieren el triunfo sino después de explorar y batir todas las fuerzas contrarias, para tenerlo de este modo más completo y permanente ${ }^{25}$.

39

\section{Universidades en que se enseña este Derecho}

La Filosofía y gusto delicado, que tanto ilustran este siglo, han hecho extender universalmente esta ciencia por toda Europa, pues no sólo florece ya en las Universidades protestantes, donde primero se introdujo como pública enseñanza, sino que tienen destinadas cátedras por los católicos en Dillinga; Friburg del Brisgoun y en Inspruk; en Viena de Austria y Praga se fundaron casi al mismo tiempo que en esta corte; y por último, se ha puesto en la Universidad de Coimbra. Por manera que en las demás capitales y provincias donde no tiene señalado magisterio, está recibido con el mismo aprecio y se estudia privadamente con singular esmero. Por cuya razón, es tanto más necesario proceder con cuidado y mucha advertencia para no deslizar en errores que, además de ser muy reprensibles, pueden traer muy fatales consecuencias.

40

No me puedo detener a referir la serie y novedades en las controversias y opiniones, las obras y juicio de los autores, las enseñanzas, sus métodos y otros puntos que pueden conducir para tener una completa noticia. Yo no me propuse dar todas las partes de que se compone la Historia Literaria de esta ciencia, sino un plan conciso que sirva de guía y norma a los que la emprenden, y juzgo haber dicho para este fin lo suficiente.

\section{FIN}

25 Véase a Finetti, De principiis juris naturae, lib. II, cap. 8. 


\section{PROGRAMA HISTORIA DEL DERECHO PUBLICACIONES}

1. Luis Grau, Origenes del constitucionalismo americano. Corpus documental bilingüe / Selected Documents Illustrative of the American Constitutionalism. Bilingual edition, 3 vols., Madrid 2009, 653+671+607 pp.

http://hdl.handle.net/10016/5669

2. Luis Grau, Nosotros el pueblo de los Estados Unidos. La Constitución de los Estados Unidos y sus enmiendas. 1787-1992. Edición bilingüe / We the People of the United States. The U.S. Constitution and its Amendments. 1787-1992. Bilingual edition, Madrid 2010, $338 \mathrm{pp.}$

http://hdl.handle.net/10016/8517

3. Carlos Petit, Fiesta y contrato. Negocios taurinos en protocolos sevillanos (1777-1847), Madrid 2011, $182 \mathrm{pp.}$

http://hdl.handle.net/10016/10145

4. Pablo Mijangos y González, El nuevo pasado jurídico mexicano. Una revisión de la historiografía jurídica mexicana durante los últimos 20 años, Madrid 2011, 110 pp.

http://hdl.handle.net/10016/10488

5. Luis Grau, El constitucionalismo americano. Materiales para un curso de historia de las constituciones, Madrid 2011, xxii+282 pp.

http://hdl.handle.net/10016/11865

6. Víctor Tau Anzoátegui, El taller del jurista. Sobre la Colección Documental de Benito de la Mata Linares, oidor, regente y consejero de Indias, Madrid 2011, $175 \mathrm{pp}$.

http://hdl.handle.net/10016/12735

7. Ramon Llull, Arte de Derecho, estudio preliminar de Rafael Ramis Barceló, traducción y notas de Pedro Ramis Serra y Rafael Ramis Barceló, Madrid 2011, 178 pp.

http://hdl.handle.net/10016/12762

8. Consuelo Carrasco García, ¿Legado de deuda? A vueltas con la Pandectística, Madrid 2011, 158 pp.

http://hdl.handle.net/10016/12823 
9. Pio Caroni, Escritos sobre la codificación, traducción de Adela Mora Cañada y Manuel Martínez Neira, Madrid 2012, xxvi + 374 pp.

http://hdl.handle.net/10016/13028

10. Esteban Conde Naranjo (ed.), Vidas por el Derecho, Madrid 2012, 569 pp. http://hdl.handle.net/10016/13565

11. Pierangelo Schiera, El constitucionalismo como discurso político, Madrid 2012, 144 pp.

http://hdl.handle.net/10016/13962

12. Rafael Ramis Barceló, Derecho natural, historia y razones para actuar. La contribución de Alasdair MacIntyre al pensamiento jurídico, Madrid 2012, 480 pp.

http://hdl.handle.net/10016/13983

13. Paola Miceli, Derecho consuetudinario y memoria. Práctica jurídica y costumbre en Castilla y León (siglos XI-XIV), Madrid 2012, 298 pp. http://hdl.handle.net/10016/14294

14. Ricardo Marcelo Fonseca, Introducción teórica a la historia del derecho, prefacio de Paolo Cappellini, Madrid 2012, 168 pp. http://hdl.handle.net/10016/14913

15. Alessandra Giuliani, Derecho dominical y tanteo comunal en la Castilla moderna, Madrid 2012, 134 pp.

http://hdl.handle.net/10016/15436

16. Luis Grau, An American Constitutional History Course for Non-American Students, Madrid 2012, $\mathrm{xx}+318 \mathrm{pp}$.

http://hdl.handle.net/10016/16023

17. Antonio Ruiz Ballón, Pedro Gómez de la Serna (1806-1871). Apuntes para una biografía jurídica, Madrid 2013, 353 pp.

http://hdl.handle.net/10016/16392

18. Tamara El Khoury, Constitución mixta y modernización en Líbano, prólogo de Maurizio Fioravanti, Madrid 2013, 377 pp.

http://hdl.handle.net/10016/16543 
19. María Paz Alonso Romero/Carlos Garriga Acosta, El régimen jurídico de la abogacía en Castilla (siglos XIII-XVIII), Madrid 2013, 337 pp.

http://hdl.handle.net/10016/16884

20. Pio Caroni, Lecciones de historia de la codificación, traducción de Adela Mora Cañada y Manuel Martínez Neira, Madrid 2013, 213 pp. http://hdl.handle.net/10016/17310

21. Julián Gómez de Maya, Culebras de cascabel. Restricciones penales de la libertad ambulatoria en el Derecho codificado español, Madrid 2013, 821 pp. http://hdl.handle.net/10016/17322

22. François Hotman, Antitriboniano, o discurso sobre el estudio de las leyes, estudio preliminar de Manuel Martínez Neira, traducción de Adela Mora Cañada, Madrid 2013, 211 pp.

http://hdl.handle.net/10016/17855

23. Jesús Vallejo, Maneras y motivos en Historia del Derecho, Madrid 2014, $184 \mathrm{pp}$.

http://hdl.handle.net/10016/1809o

24. María José María e Izquierdo, Los proyectos recopiladores castellanos del siglo XVI en los códices del Monasterio de El Escorial, Madrid 2014, $248 \mathrm{pp}$.

http://hdl.handle.net/10016/18295

25. Regina Polo Martín, Centralización, descentralización y autonomía en la España constitucional. Su gestación y evolución conceptual entre 1808 y 1936, Madrid 2014, 393 pp.

http://hdl.handle.net/10016/18340

26. Massimo Meccarelli/Paolo Palchetti/Carlo Sotis (eds.), Il lato oscuro dei Diritti umani: esigenze emancipatorie e logiche di dominio nella tutela giuridica dell'individuo, Madrid 2014, 390 pp.

http://hdl.handle.net/10016/18380

27. María López de Ramón, La construcción histórica de la libertad de prensa: Ley de policía e imprenta de 1883, Madrid 2014, 143 pp.

http://hdl.handle.net/10016/19296 
28. José María Coma Fort, Codex Theodosianus: historia de un texto, Madrid 2014, 536 pp.

http://hdl.handle.net/10016/19297

29. Jorge Alberto Núñez, Fernando Cadalso y la reforma penitenciaria en España (1883-1939), Madrid 2014, 487 pp.

http://hdl.handle.net/10016/19662

30. Carlos Petit, Discurso sobre el discurso. Oralidad y escritura en la cultura jurídica de la España liberal, Madrid 2014, 185 pp.

http://hdl.handle.net/10016/19670

31. Jean-Étienne-Marie Portalis, Discurso preliminar sobre el proyecto de Código civil, Madrid 2014, 53 pp.

http://hdl.handle.net/10016/19797

32. Cesare Beccaria, Tratado de los delitos y de las penas, Madrid 2015, $87 \mathrm{pp}$. http://hdl.handle.net/10016/20199

33. Massimo Meccarelli y Paolo Palchetti (eds.), Derecho en movimiento: personas, derechos y derecho en la dinámica global, Madrid 2015, 256 pp. http://hdl.handle.net/10016/20251

34. Alessandro Somma, Introducción al derecho comparado, traducción de Esteban Conde Naranjo, Madrid 2015, 193 pp.

http://hdl.handle.net/10016/20259

35. A. F. J. Thibaut, Sobre la necesidad de un derecho civil general para Alemania, Madrid 2015, $42 \mathrm{pp}$.

http://hdl.handle.net/10016/21166

36. J.-J.-R. de Cambacérès, Discursos sobre el Código civil, Madrid 2015, 61 pp. http://hdl.handle.net/10016/21254

37. Ramon Llull, Arte breve de la invención del derecho, estudio preliminar de Rafael Ramis Barceló, traducción de Pedro Ramis Serra y Rafael Ramis Barceló, Madrid 2015, 233 pp.

http://hdl.handle.net/10016/21406 
38. F. C. von Savigny, De la vocación de nuestra época para la legislación y la ciencia del Derecho, Madrid 2015, 130 pp.

http://hdl.handle.net/10016/21520

39. Joaquín Marín y Mendoza, Historia del derecho natural y de gentes, Madrid 2015, $40 \mathrm{pp}$.

http://hdl.handle.net/10016/22079 